\title{
Caracterización de uniones soldadas robotizadas GTAW-P sin metal de aporte en acero inoxidable ferrítico AISI 430
}

\author{
Graciela Rosel $^{\mathrm{a}}$, Benjamín Vargas ${ }^{\mathrm{a}}$, Verónica Estrella ${ }^{\mathrm{a}}$, Eva Cervantes ${ }^{\mathrm{b}}$, Jaime Taha $^{\mathrm{c}}$, Celso Cruz $^{\mathrm{d}}$ \\ ${ }^{a}$ Tecnológico Nacional de México / IT de Tlalnepantla, División de Estudios de Posgrado e Investigación, Av. Instituto Tecnológico s/n, \\ 54070 Col. La comunidad, Tlalnepantla de Baz, Estado de México, México. \\ ${ }^{b}$ METALSA México, Parque de Investigación e Innovación Tecnológica (PIIT), Apodaca Nuevo León, México. \\ ${ }^{c}$ Universidad de Monterrey, Departamento de Ingeniería, San Pedro Garza García, N.L, México. Departamento de Tecnología de \\ METALSA, Parque de Investigación e Innovación Tecnológica (PIIT), Apodaca Nuevo León, México. \\ ${ }^{d}$ CIDESI Estado de México, Av. Desarrollo s/n Parque Industrial Cuamatla, 54763 Cuautitlán Izcalli, México.
}

*grace.ros10@gmail.com

\section{Resumen}

Se estudió el efecto de la corriente pulsada y velocidad de avance de soldadura sobre la resistencia mecánica bajo carga de tensión, zonas microestructurales, tamaño de grano y número de tamaño de grano ASTM G en la Zona Afectada por el Calor (ZAC) de uniones soldadas robotizadas GTAW-P por fusión en lámina de acero inoxidable ferrítico AISI 430 con $1.5 \mathrm{~mm}$ de espesor. Para la caracterización mecánica se usaron pruebas de tracción para evaluar la resistencia a la tensión. Se aplicó microscopía óptica para la evolución microestructural y procesamiento digital de imágenes (PDI) para la obtención de micrografías panorámicas de amplio formato de uniones transversales. Los resultados sugirieron que la condición óptima (JR1) fue la combinación de la corriente de soldadura $(61 / 83.9$ A), arco pulsado $(15 \mathrm{~Hz})$ y velocidad de soldadura (381 $\mathrm{mm} / \mathrm{min}$ ), lo que resultó en la fusión completa del metal con geometría adecuada del cordón, resistencia máxima a la tracción de $457 \mathrm{MPa}$, tamaño de grano mediano $(10.84 \mu \mathrm{m})$, ferrita aliotromórfica dentro de la ZAC recristalizada y ferrita tipo placa Widmanstätten secundaria dentro del metal fundido.

Palabras clave: Acero inoxidable ferrítico 430, soldadura robotizada GTAW-P, micrografias panorámicas, tamaño de grano.

\section{Introducción}

El acero inoxidable ferrítico (AIF) es una aleación que comúnmente tiene 16 a $18 \% \mathrm{Cr}$, puede ser laminada en frío o caliente y posee alta ductilidad que se obtiene del proceso de recocido [1]. Las aplicaciones comerciales de este acero IF 430 se encuentran en diversos componentes como escapes automotrices, electrodomésticos, conductos, campanas de cocina y recubrimientos de tanques [2] [3] [4]. En la actualidad, el costo del acero inoxidable austenítico ha aumentado debido a la volatilidad del costo del $\mathrm{Ni}$, que está presente en su composición química, derivado de ello, el acero IF se han convertido en alternativa asequible. Uno de los principales inconvenientes del acero IF es su limitada soldabilidad ya que, durante la soldadura, existe transformación de fase $\mathrm{Y}\left(917^{\circ} \mathrm{C}\right)$, crecimiento de grano $\left(927{ }^{\circ} \mathrm{C}\right)$, martensita intergranular o en islas en la ZAC y cordón de soldadura. Otro desafío de este acero es la falta de penetración completa de la soldadura. Estos fenómenos afectan su resistencia a la tracción y disminuyen su elongación [5] [6].
Existen múltiples investigaciones al respecto, como el estudio de Amuda et al. [3] quienes documentaron la influencia del calor de entrada y velocidad de soldadura en Acero IF AISI 430 de $1.5 \mathrm{~mm}$ de espesor mediante proceso GTAW automatizado sin metal de aporte y soldadura convencional, para compararlas con la aplicación de $\mathrm{Ti}$ y $\mathrm{Al}$ adicionadas en polvo. Determinaron que a $0.500 \mathrm{~kJ} / \mathrm{mm}$, la susceptibilidad al proceso de sensibilización se acelera, modificando la microestructura, tamaño de grano y afectando la resistencia del componente soldado en servicio. En ese sentido, Amuda y Mridha [7] indagaron sobre el proceso GTAW evaluando la velocidad de soldadura $(120$ a $180 \mathrm{~mm} / \mathrm{min})$ y corriente (70 a 110 A) para el acero IF 430, obteniendo tamaños de grano ferrítico de 30 a $37 \mu \mathrm{m}$, producidos por valores de calor de entrada neto $\left(Q_{\text {net }}\right)$ de 0.336 a 0 . $634 \mathrm{~kJ} / \mathrm{mm}$. Por otro lado, Giridharan y Murugan [6] aplicaron la metodología de superficie de respuesta para optimizar la geometría del cordón y penetración de soldadura en cupones de acero inoxidable AISI 304L mediante el proceso de soldadura GTA pulsado donde evaluaron la corriente pulsada (180 a 220 A), duración del 
pulso (450 a $650 \mathrm{~ms}$ ) y velocidad de soldadura (110 a $190 \mathrm{~mm} / \mathrm{min})$, colocando electrodo de $\mathrm{W}$ a $60^{\circ}$, utilizando gas $\mathrm{Ar}$ con caudal de 10 y $5 \mathrm{~L} / \mathrm{min}$ para protección y respaldo, respectivamente. Determinaron la combinación óptima de $211.4 \mathrm{~A}$, $537.650 \mathrm{~ms}$ y $165.1 \mathrm{~mm} / \mathrm{min}$, logrando penetración de $3.4 \mathrm{~mm}$ y área de cordón de $17.89 \mathrm{~mm}^{2}$.

Debido a los desafíos de la soldabilidad robotizada en acero IF, es necesario integrar mediante el análisis y estudio de distintas técnicas eficientes que permitan reducir el costo de la soldadura en este tipo de acero sin comprometer la calidad e integridad mecánica de juntas en componentes estructurales soldados. El presente trabajo se centró en caracterizar el efecto de la corriente, arco pulsado y velocidad de soldadura del proceso soldadura GTAW-P robotizada sin metal de aporte en corridas experimentales sobre lámina de acero inoxidable ferrítico AISI 430.

\section{Experimentación}

\subsection{Materiales}

Las láminas de acero IF utilizadas para el estudio tenían $1000 \times 1000 \times 1.5 \mathrm{~mm}$ de largo, ancho $\mathrm{y}$ espesor, respectivamente [8]. Estas se caracterizaron química y mecánicamente para obtener una identificación del acero, así como documentar los cambios en la resistencia mecánica bajo tensión.

\subsection{Caracterización química}

Se realizó análisis químico por emisión óptica sobre muestras de $25 \times 25 \times 1.5 \mathrm{~mm}$ mediante espectrómetro SPECTRO. El procedimiento experimental se basó en estándar ASTM E353 [9]. De acuerdo con Guiraldenq y Hardouin [10] se calcularon el $\mathrm{Cr}$ y $\mathrm{Ni}$ equivalentes con ecuaciones 1 y 2, cuyos resultados corresponden a la predicción de microestructura que se generase en proceso de soldadura sobre la matriz ferrítica.

$$
\begin{aligned}
& C r_{e q}=C r \%+M o \%+1.5 S i \%+0.5 C b \% \\
& N i_{e q}=N i \%+30 C \%+0.5 M n \%
\end{aligned}
$$

Los parámetros explorados para el presente análisis provienen de la literatura y experimentación previa [3] [7] [11] [12] [13] [14] sustentado un diseño de experimentos, de donde se obtuvieron las combinaciones de parámetros. Teniendo como variables exógenas: corriente de soldadura (base y pico, dentro del rango 47 a $61 \mathrm{~A}$ / 63 a $84 \mathrm{~A}$, respectivamente), velocidad de soldadura (330 a $381 \mathrm{~mm} / \mathrm{min}$ ) y pulsos del arco
(10 a $18.4 \mathrm{~Hz})$. Las combinaciones caracterizadas se muestran en Tabla 1 .

Tabla 1. Combinaciones de parámetros de soldadura robotizada GTAW-P en acero IF 430.

\begin{tabular}{|c|c|c|c|}
\hline Unión & $\begin{array}{c}\text { Corriente de } \\
\text { soldadura } \\
\text { (A) }\end{array}$ & $\begin{array}{c}\text { Velocidad de } \\
\text { soldeo } \\
\text { (mm/min) }\end{array}$ & $\begin{array}{c}\text { Pulsos } \\
\text { del arco } \\
\text { (Hz) }\end{array}$ \\
\hline JR1 & $61 / 84$ & 381 & 15 \\
\hline JR2 & $52 / 99.5$ & 330.2 & 10 \\
\hline JR3 & $50 / 63$ & 330.2 & 18.4 \\
\hline
\end{tabular}

\subsection{Proceso de soldadura}

Los cupones de soldadura se maquinaron con dimensiones de $100 \times 70 \times 1.5 \mathrm{~mm}$, su preparación consistió en desbaste con papel abrasivo de óxido de aluminio grado 300 y limpieza con acetona industrial. El diseño de junta fue bisel recto sin apertura de raíz [13] con posición $1 \mathrm{G}$, ángulo de antorcha de $90^{\circ}$, electrodo W-Th2\% y un solo paso de soldadura, como se puede ver en figura 1 . Se utilizó robot de soldadura Fanuc Arc mate 100iC y fuente de energía eléctrica de Lincoln (Power Wave S500) con gas de protección de $99.99 \% \mathrm{Ar}$ a $12 \mathrm{~L} /$ min y sin gas de respaldo.

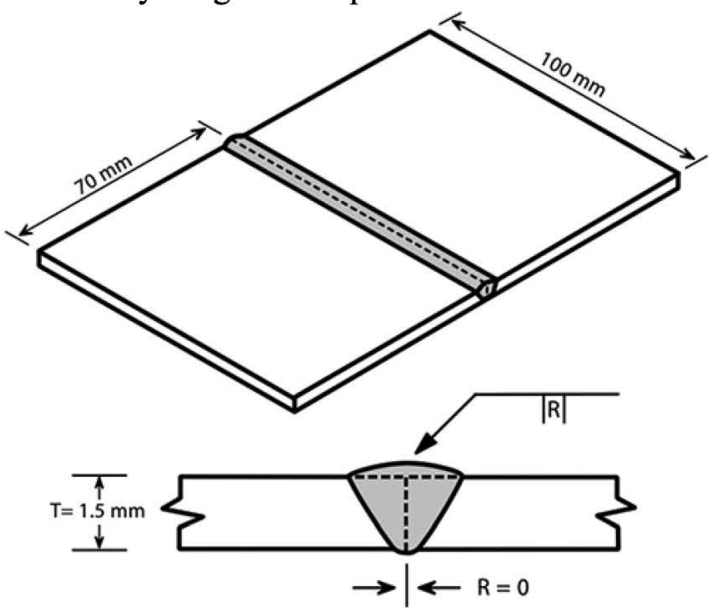

Figura 1. Diseño de unión soldada para acero inoxidable ferrítico sin apertura de raíz.

El cálculo del calor de entrada $\left(\mathrm{Q}_{\text {net }}\right)$ se basó en literatura especializada [15] [16] como se muestra en ecuaciones 3 y 4 , con eficiencia de transferencia de calor de 0.85 .

$$
Q_{\text {arc }}=60 \frac{w_{c} \cdot w_{v}}{w_{t s}},
$$


Donde $Q_{a r c}$ es energía del arco, $W C$ es corriente de soldadura, $W_{v}$ es voltaje, y $W_{t s}$ es velocidad de soldeo.

$$
Q_{\text {net }}=\eta Q_{\text {arc }}
$$

Donde $Q_{n e t}$ es calor de entrada $(\mathrm{kJ} / \mathrm{mm})$, y $\eta_{\text {es eficiencia del arco. }}$

\subsection{Caracterización mecánica}

La determinación de la resistencia mecánica se realizó siguiendo estándar ASTM E8 [17]. Las probetas se obtuvieron del centro de lámina bajo procedimiento ASTM A370 [18]. Las muestras se mecanizaron utilizando máquina de corte por chorro de agua Mitsubishi Electric Supreme, obteniendo probetas de tensión reducidas con 6 $\mathrm{mm}$ de ancho y $25 \mathrm{~mm}$ de longitud calibrada, para posteriormente ensayarlas en máquina electromecánica Instron 4482. La rapidez de deformación fue $8 \mathrm{MPa}$ y $15 \mathrm{~mm} / \mathrm{min}$ para offset del $0.2 \%$ y resistencia a la tensión, respectivamente. La resistencia a la cedencia $\left(\mathrm{S}_{0}\right)$ se calculó utilizando extensómetro de clase B-1 de $50 \mathrm{~mm}$ de longitud de calibre. La elongación después de fractura se determinó con longitud calibrada de $50 \mathrm{~mm}$ marcada en superficie de cada muestra. Se evaluaron dos probetas de tensión por combinación de parámetros y se informaron resultados promedio.

\subsection{Caracterización microestructural}

Para el análisis mediante microscopía óptica de uniones soldadas, se utilizaron muestras de sección transversal tomadas del centro de cupones con dimensiones de $22 \times 1.5 \mathrm{~mm}$ de largo y espesor, respectivamente. Se empleó el procedimiento ASTM E3 [19] para metalografía, iniciando con encapsulado en baquelita de muestras, posteriormente se desbastaron con papel abrasivo de $\mathrm{SiC}$ grado 240 hasta 2000 , para pulirlas con pasta de diamante de 5 y $7 \mu \mathrm{m}$ y finalmente hacer ataque químico con reactivo Vilella para revelar las características microestructurales para 50 a $500 \mathrm{x}$ mediante observaciones realizadas en microscopio óptico Axiovert 40 MAT con cámara Axio Vert ERc 5s Carl Zeiss. La determinación del número de tamaño de grano ASTM G y tamaño de grano promedio, se realizó bajo metodología de tres círculos de Abrams de acuerdo con ASTM E112 [20] utilizando seis campos por muestra evaluada a 200x. Este método permitió la cuantificación estandarizada del número ASTM G, es decir, tamaño de micro-granos por pulgada cuadrada, reduciendo error mediante mediciones aleatorias. Se utilizó procesamiento digital de imágenes para generar micrografías transversales panorámicas utilizando de seis a ocho campos a 50x.

\section{Resultados y discusiones}

\subsection{Análisis químico}

Los resultados arrojaron que el acero en condición original, presento correspondencia al estándar ASTM A240 [8] y derivado de estos elementos químicos, las microestructuras generadas en el proceso de soldadura se enmarcarían en fases de ferrita y martensita, hecho que se comprobó mediante análisis microestructural.

Tabla 2. Composición química del acero inoxidable AISI 430 (\% peso).

\begin{tabular}{|l|l|l|l|l|l|l|l|l|}
\hline $\mathbf{F e}$ & $\mathbf{C}$ & $\mathbf{S i}$ & $\mathbf{M n}$ & $\mathbf{C r}$ & $\mathbf{N i}$ & $\mathbf{P}$ & $\mathbf{C r}_{\text {eq }}$ & $\mathbf{N i}_{\text {eq }}$ \\
\hline 82.32 & 0.03 & 0.23 & 0.49 & 16.16 & 0.22 & 0.03 & 16.5 & 1.3 \\
\hline
\end{tabular}

\subsection{Resistencia mecánica bajo tracción}

Después de analizar los resultados de propiedades mecánicas de resistencias máxima a la tensión $\left(\mathrm{S}_{\mathrm{u}}\right)$ y a la cedencia $\left(\mathrm{S}_{0}\right)$, así como elongación de uniones soldadas por técnica GTAW-P robotizada sin metal de aporte, se compararon con estándar ASTM A240 [8], destacando dos corridas (JR1 y JR2) que superaron mínimo requerido $(450 \mathrm{MPa})$ para $\mathrm{S}_{\mathrm{u}}$ como se puede ver en tabla 3 , que muestra tres pruebas robotizadas cuyo rango de resistencia alcanzado fue 440 a $457 \mathrm{MPa}$.

Tabla 3. Propiedades mecánicas bajo tensión de uniones soldadas GTAW-P robotizadas en acero inoxidable 430.

\begin{tabular}{|c|c|c|c|c|c|c|}
\hline \multirow{2}{*}{$\begin{array}{c}\text { Condi } \\
\text { ción }\end{array}$} & \multicolumn{3}{|c|}{$\begin{array}{l}\text { Combinación de } \\
\text { parámetros }\end{array}$} & \multirow{2}{*}{$\begin{array}{c}\mathbf{S}_{\mathbf{u}} \\
(\mathbf{M P} \\
\mathbf{a})\end{array}$} & \multirow{2}{*}{$\begin{array}{c}\mathrm{S}_{\mathbf{0}} \\
0.2 \% \\
\text { (MPa) }\end{array}$} & \multirow{2}{*}{$\begin{array}{c}\begin{array}{c}\text { Elonga } \\
\text { ción }\end{array} \\
(\%)\end{array}$} \\
\hline & $\begin{array}{c}\begin{array}{c}\text { Corrie } \\
\text { nte }\end{array} \\
\text { (A) }\end{array}$ & $\begin{array}{c}\text { Veloci } \\
\text { dad } \\
(\mathbf{m m} / \\
\text { min) }\end{array}$ & $\begin{array}{c}\begin{array}{c}\text { Puls } \\
\text { os }\end{array} \\
\text { (Hz) }\end{array}$ & & & \\
\hline JR1 & $61 / 84$ & 381 & 15 & 457 & 333 & 31 \\
\hline JR2 & $\begin{array}{l}521 \\
99.5\end{array}$ & 330.2 & 10 & 452 & 331 & 28 \\
\hline JR3 & $50 / 63$ & 330.2 & 18.4 & 440 & 310 & 30 \\
\hline
\end{tabular}




\begin{tabular}{|c|l|l|l|l|}
\hline $\begin{array}{c}\text { Acero } \\
\text { IF }\end{array}$ & & 498 & 348 & 32 \\
\hline
\end{tabular}

El comportamiento típico de tracción axial expresado por curvas esfuerzo - deformación para tres uniones (JR1, JR2 y JR3) se pueden observar en figura 2, que expresa el incremento en valores de $\mathrm{S}_{\mathrm{u}}$ de acuerdo con las combinaciones de parámetros de soldadura aplicada. La unión con mejor rendimiento fue JR1 que muestra los más altos valores de $\mathrm{S}_{\mathrm{u}} \mathrm{y}$ elongación, como resultado de la mejor selección de parámetros $(61 / 84$ A, 381 $\mathrm{mm} / \mathrm{min}$ y $15 \mathrm{~Hz}$ ) con $\mathrm{Q}_{\text {net }}$ de $0.168 \mathrm{~kJ} / \mathrm{mm}$, cuya $\mathrm{S}_{\mathrm{u}}$ excede margen de seguridad del estándar ASTM A240 [8]. Resultados similares fueron reportados por Gurrama et al. [14] como valor óptimo (457 MPa) para $\mathrm{S}_{\mathrm{u}}$ en este tipo de juntas soldadas. Sin embargo, hubo disminuciones de $8 \%$ para $S_{u}, 4 \%$ de $S_{0}$ y 3\% para elongación, en comparación con propiedades del acero inoxidable 430 en condición original.

La $\mathrm{S}_{0}$ de corridas mencionadas, se definió claramente para las tres combinaciones de parámetros (ver cuadro insertado en gráfica) desde la zona de transición entre comportamiento elástico-plástico, resultando que junta JR1 exhibió la mayor resistencia a la cedencia, seguida de uniones JR2 y JR3 con resistencia inferior asociada con la combinación: $50 / 63 \mathrm{~A}, 18.4 \mathrm{~Hz}$ y $330.2 \mathrm{~mm} / \mathrm{min}$.

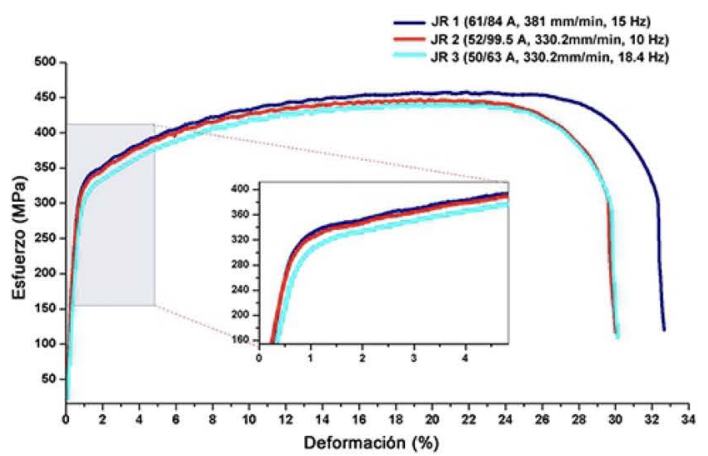

Figura 2. Curva esfuerzo - deformación para tres condiciones de soldadura robotizada GTAW-P sin metal de aporte.

\subsection{Evolución microestructural}

El cambio en características microestructurales varió de acuerdo con la combinación de parámetros de soldadura robotizada en cada unión. El recorrido JR1 en vista panorámica transversal muestra varios granos gruesos bien definidos dentro de la ZAC y granos más grandes en metal fundido (figura 3a). La ZAC recristalizada exhibió granos gruesos ferríticos cerca de la línea de fusión, además de granos de tamaños medianos y finos cerca del metal base. Con más detalle, se encuentra la baja presencia de ferrita Widmanstätten secundaria en placa (SWSF) y ferrita aliotromórfica (ALF) dentro de límites de grano [21] debido al cambio de fase durante la soldadura y solidificación del metal [6], como puede ser visto en figura $3 \mathrm{~b}$ ). El metal fundido o cordón de soldadura, mostró fases de ferrita Widmanstätten en placa lateral (WFSP), ferrita acicular (AF) y ferrita aliotromórfica de límite de grano (GBFA) en base a lo informado por Tomasz et al. [22], Además, hay martensita "peppery" como isla gris [23] (figura 3c). Finalmente, el metal base está compuesto por matriz de granos ferríticos que muestran diferentes tamaños y orientaciones que siguen la dirección del laminado (figura 3d). Por lo tanto, este comportamiento microestructural indujo resistencia mecánica superior de unión soldada JR1, tales como la $S_{u}$ y $S_{0}$. Esta correlación entre las zonas microestructurales y propiedades mecánicas se favoreció con $Q_{\text {net }}$ adecuado $(0.168$ $\mathrm{kJ} / \mathrm{mm}$ ) derivado de la mejor combinación de parámetros de soldadura robotizada $(61 / 84 \mathrm{~A}, 15$ $\mathrm{Hz}$ y $381 \mathrm{~mm} / \mathrm{min}$ ).

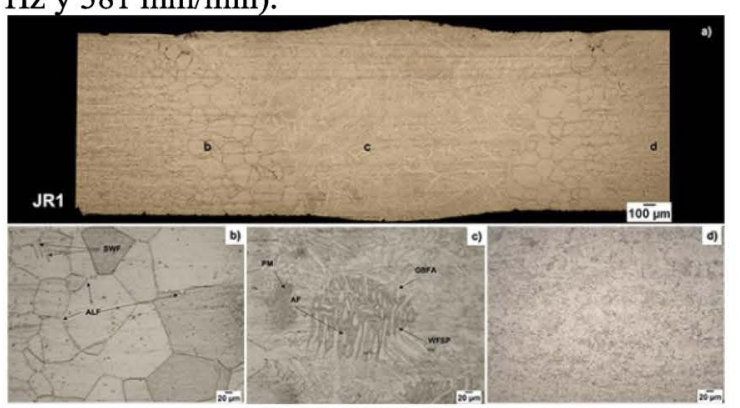

Figura 3. Micrografías obtenidas a través de microscopía óptica de junta soldada JR1 robotizada GTAW-P: a) dos zonas microestructurales en junta, b) ferrita aliotromórfica en ZAC, c) ferrita de placa lateral Widmanstätten en metal fundido y d) matriz ferrítica en metal base.

En unión JR2 transversal se observan claramente granos columnares alargados en dirección del flujo de calor hacia el centro de junta, esto se debe al proceso de enfriamiento durante la solidificación, lo que resulta en cordón de soldadura. La ZAC muestra granos ferríticos con crecimiento notable en ambos lados de unión (figura 4a) con respecto al metal base, así como los granos exhibieron diferentes tamaños. La ZAC presentó ferrita poligonal intragranular en límites de grano (IPF), así como martensita intergranular (IM) similar a la reportada por Amuda [6], ver figura 4b). El metal de fusión (figura 4c) mostró 
ferrita columnar a partir de la cual crecen placas laterales de ferrita Widmanstätten (WFSP), ferrita poligonal intragranular (IPF), ferrita acicular (AF) y martensita "peppery" en mayor cantidad que la reportada por Devendranath et al. [23] y que ha sido documentado por Lippold y Kotecky afirmando que la martensita es transformación de austenita formada en alta temperatura durante el enfriamiento y precipitación de carburos (C) [24]. El metal base presento matriz ferrítica con granos de diferentes tamaños (figura 4d). Estas fases microestructurales complejas de la ZAC y metal fundido se produjeron con valor $\mathrm{Q}_{\text {net }}$ más alto $(0.242 \mathrm{~kJ} / \mathrm{mm})$ resultante de parámetros de soldadura $(52 / 99.5 \mathrm{~A}, 10 \mathrm{~Hz}$ y $330.2 \mathrm{~mm} / \mathrm{min})$. Finalmente, esta unión de soldadura robotizada JR2 alcanzó segundo valor de $S_{u}$ como consecuencia de fases microestructurales explicadas para la ZAC y metal fundido.

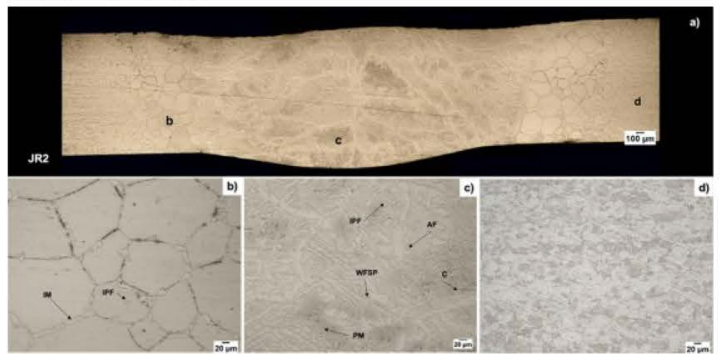

Figura 4. Micrografías obtenidas por microscopía óptica de unión soldada JR2 robotizada GTAW-P: a) granos columnares en metal fundido, b) martensita intergranular en ZAC, c) placas laterales de ferrita Widmanstätten en metal fundido y d) granos ferríticos en metal base.

La condición JR3 muestra simetría en geometría del cordón fundido con granos ferríticos, y la ZAC exhibe granos gruesos con diferentes tamaños (figura 5a). La ZAC recristalizada presenta granos gruesos de ferrita que contienen ferrita aliotromórfica (ALF) en límites de grano y martensita intergranular (IM), ambos en cantidad menor que la del ensayo JR2, como se ve en figura $5 b)$. El cordón de soldadura se caracteriza por estructuras como placas laterales de ferrita Widmanstätten intragranular (WFSP), ferrita poligonal, ferrita primaria poligonal no alineada (NPPF) [21], así como islas de martensita (M) [6] [7]. En cuanto al metal base está formado por granos ferríticos. Estas microestructuras de soldadura JR3 se generaron por $\mathrm{Q}_{\text {net }}$ de 0.158 $\mathrm{kJ} / \mathrm{mm}$, resultante de parámetros de soldadura $(50 / 63 \mathrm{~A}, 18.4 \mathrm{~Hz}$ y $330.2 \mathrm{~mm} / \mathrm{min})$, que explicaron su $\mathrm{S}_{\mathrm{u}}$ inferior a la de condiciones JR1 y JR2.

La caracterización cuantitativa de la ZAC consistió en dos parámetros: tamaño de grano y número de tamaño de grano ASTM G para soldaduras robotizadas GTAW-P sin metal de aporte.

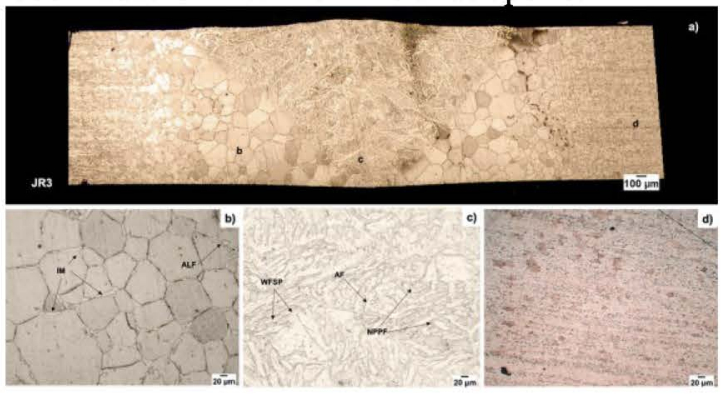

Figura 5. Micrografías obtenidas por microscopía óptica de unión JR3 robotizada GTAW-P: a) granos gruesos en $\mathrm{ZAC}, \mathrm{b}$ ) ferrita aliotromórfica en ZAC, c) ferrita acicular en metal fundido y d) matriz ferrítica para metal base.

\subsection{Tamaño de grano y Número G ASTM}

La figura 6 muestra el tamaño de grano promedio y número de tamaño de grano ASTM G dentro de la ZAC con respecto al $\mathrm{Q}_{\text {net, }}$ donde es notable que hubo dos cambios significativos. El tamaño de grano alcanzado por unión JR2 fue 7.42 $\mu \mathrm{m}$, mientras que número ASTM G resultó de 11.2. Es evidente que el comportamiento del número $G$ es inversamente proporcional a valores de tamaño de grano [36], lo que afecta las propiedades mecánicas, como lo demuestran Amuda y Mridha [6].

Con respecto a corrida JR1 mostro tamaño de grano promedio de $10.84 \mu \mathrm{m}$ y G de 10.1, ambos cercanos al valor mínimo de rangos reportados [20], lo que corroboró el mayor rendimiento mecánico de tracción con $\mathrm{Q}_{\text {net }}$ más bajo que reportado en literatura [3] [6] [7]. La condición JR3 está en primer lugar con $Q_{\text {net }}$ de $0.158 \mathrm{~kJ} / \mathrm{mm}$, tamaño de grano de $13.82 \mu \mathrm{m}$, es decir, 4.8 veces más grande que metal base, además, mostró fusión y penetración completas con $\mathrm{S}_{\mathrm{u}}$ de $440 \mathrm{MPa}$.

Por lo tanto, la ZAC de corrida JR2 (52 / 99.5 A, $10 \mathrm{~Hz}$ y $330.2 \mathrm{~mm} / \mathrm{min}$ ) exhibió tamaño de grano más bajo $(7.42 \mu \mathrm{m})$ y segundo lugar del valor $\mathrm{S}_{\mathrm{u}}$. Todas estas propiedades mecánicas críticas $\mathrm{y}$ parámetros microestructurales se vincularon a fase endurecida como martensita en límites de grano. Este comportamiento se relacionó con mayor $\mathrm{Q}_{\text {net }}(0.242 \mathrm{~kJ} / \mathrm{mm})$ con aplicación de corriente pulsada, causando distorsión térmica (deformación) en ambas láminas de unión soldada y geometría del cordón. 
Unión soldada

31

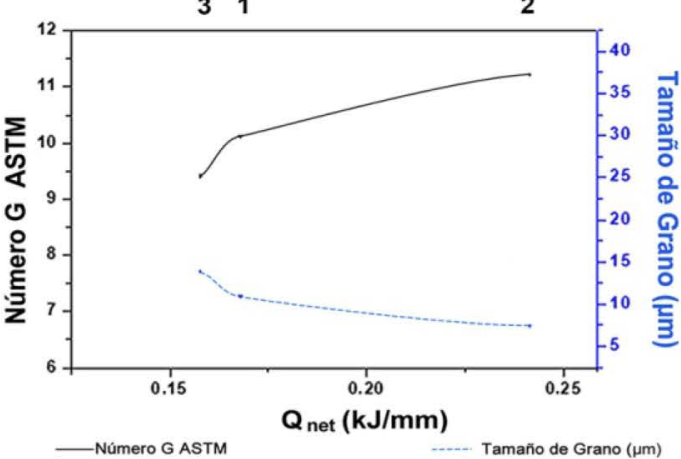

Figura 6. Variación del tamaño de grano y número $G$ ASTM en relación con calor de entrada en uniones soldadas GTAW-P robotizadas.

\section{Conclusiones}

Después de la caracterización mecánica, microestructural y análisis de resultados experimentales, la combinación óptima de parámetros de soldadura por fusión para uniones soldadas GTAW pulsada robotizada, fue condición JR1. Los parámetros de soldadura JR1 incluyeron corriente de soldadura pico y base de 61/84 A, frecuencia del arco pulsado de $15 \mathrm{~Hz}$ y 381 $\mathrm{mm} / \mathrm{min}$ de velocidad de soldadura. Esta corrida JR1 alcanzo fusión y penetración completas en metal fundido, con mejor resistencia mecánica bajo tensión sin utilizar metal de aporte, es decir, resistencia máxima a la tracción de $457 \mathrm{MPa}$, resistencia a la cedencia y elongación dentro del estándar y cercanos a propiedades del acero inoxidable 430 como fue recibido. Estas propiedades fueron vinculadas a fases microestructurales de ferrita aliotromórfica en la ZAC recristalizada y presencia de ferrita Widmanstätten secundaria de placa lateral dentro del metal fundido. Este comportamiento mecánico y microestructural se vinculó al calor de entrada neto de $0.168 \mathrm{~kJ} / \mathrm{mm}$.

\section{Agradecimientos}

Este proyecto es fruto de la adición de la academia e industria, fue posible gracias al Consejo Nacional de Ciencia y Tecnología (CONACyT), METALSA, Lincoln Electric Welding School, Centro de Ingeniería y Desarrollo Industrial (CIDESI), EMCO TEST, así como todas y cada una de las personas que colaboraron y a quienes agradecemos su desempeño. Reconocemos con amplia gratitud a Dra. G. Palacios, G. Bañuelos, a los Ingenieros Raúl Vázquez, A. Gómez, S. Martínez, y A. Zamora.

\section{Referencias}

1. AWS, Welding Handbook 9.4, EUA: AWS, 2011.

2. E. Bayraktar, J. Moiron, D. Kaplan, "Effect of welding conditions on the formability characteristics of thin sheet steels: Mechanical and metallurgical effects," Journal of Materials Processing Technology, vol. 175, pp. 20-26, 2006. doi:10.1016/j.jmatprotec.2005.04.007

3. M.O.H Amuda, E. T. Akinlabi, S. Mridha, "Influences of Energy Input and Metal Powder Addition on Carbide Precipitation in AISI 430 Ferritic Stainless Steel Welds," Materials Today, vol. Proceedings 4, pp. 234-243, 2017.

4. YOU Xiang-mi, JIANG Zhou-hua, LI Hua-bing "Ultra-Pure Ferritic Stainless Steels - Grade Refining Operation and Application," Journal of Iron and Steel Research International, vol. 14, $\mathrm{n}^{\circ} 4$, pp. 24-20, 2007. doi.org/10.1016/S1006-706X(07)60053-3

5. P. Giridharan, N. Murugan, "Optimization of pulsed GTA welding process parameters for the welding of AISI 304L stainless steel sheets," Advanced Manuf Technol, vol. 40, pp. 478-489, 2009.

6. M.O.H. Amuda, S. Mridha, "Comparative evaluation of grain refinement in AISI 430 FSS welds by elemental metal powder addition and cryogenic cooling," Materials and Design, vol. 35, pp. 609-618, 2012 . doi:10.1016/j.matdes.2011.09.066

7. M.O.H. Amuda, S. Mridha, "Grain refinement and hardness distribution in cryogenically cooled ferritic stainless steel welds," Materials and Design, vol. 47, pp. 365-371, 2013. dx.doi.org/10.1016/j.matdes.2012.12.008

8. ASTM, A240/A240M - 14 Standard Specification for Chromium and Chromium-Nickel Stainless Steel Plate,Sheet, and Strip for Pressure Vessels and for General Applications, West Conshohocken, PA: ASTM International, 2014

9. ASTM E353, Standard Test Methods for Chemical Analysis of Stainless, Heat-Resisting, Maraging, and Other Similar Chromium-Nickel-Iron Alloys, West Conshohocken, PA: DOI: 10.1520/E0353-14, 2014.

10. P. Guiraldenq, O. Hardouin-Duparc, "The genesis of the Schaeffler diagram in the history of stainless," Metallurgical Research \& Technology, pp. 114, 613, 2017.

11. M. Gurrama, A. Kumar, P. Ravinder, G. Madhusudhan, "Effect of copper and aluminium addition on mechanical properties and corrosion behaviour of AISI 430 ferritic stainless steel gas tungsten arc welds," journal of materials reseach and technology, vol. 2, n⿳ 3, pp. 238-249, 2013.

12. Akhilesh Kumar Singh, Vidyut Dey, Ram Naresh Rai "Techniques to improveweld penetration in TIG 
welding (A review)," Materials Today: Proceedings, Vols.4 Issue 2, Part A, pp. Pages 1252-1259, 2017.

13. AWS, D 1.6 Structural Welding Code-Stainless Steel, Miami, FL: American Welding Society, 2007.

14. M. Gurrama , A. Kumar P. Ravinder Reddy , G. Madhusudhan Reddy, , "Influence of grain refining elements on mechanical properties of AISI 430 ferritic stainless steel weldments - Taguchi approach,", Materials \& Design, vol. 36, pp. 443450, 2012.

15. AWS, Welding Inspection Technology, Miami, Florida: AWS, 2005.

16. I. Guzman-Flores, B. Vargas-Arista, J. GascaDominguez, C. Cruz-Gonzalez, M. GonzálezAlbarrán, J. del Prado-Villasana, "Effect of Torch Weaving on the Microstructure, Tensile and Impact Resistances, and Fracture of the HAZ and Weld Bead by Robotic GMAW Process on ASTM A36 Steel," Soldagem \& Inspeção, vol. 22, n 1, pp. 72-86, 2017. doi.org/10.1590/0104-9224/SI2201.08

17. ASTM, E-8 -E8M Standard Test Methods for Tension Testing of Metallic Materials, West Conshohocken: United States.

18. ASTM, A370 Standard Test Methods and Definitions for Mechanical Testing of Steel Products, West Conshohocken: ASTM International.

19. ASTM, E-3 Standard Guide for Preparation of Metallographic Specimens, West Conshohocken, EU: ASTM International, 2011.

20. ASTM, E112-96 Standard test methods for determining average grain size, Philadelphia: ASTM, 2004.

21. G. Thewlis, "Classification and quantification of microstructures in steels," Materials Science and Technology, vol. 161, $\mathrm{n}^{\circ} \quad 20$ DOI 10.1179/026708304225010325, pp. 143, 2004.

22. Tomasz, Węgrzyn, Piwnik, J. Stali, Spawanie, Chłodzeniem, Mikrojetowym., "Low Alloy Steel Welding with Micro-Jet Cooling.," Archives of Metallurgy and Materials., vol. 57, $\mathrm{n}^{\mathrm{o}}$ 2, pp. doi 10.2478/v10172-012-0056-x., 2012.

23 K. Devendranath Ramkumara, Aditya Chandrasekhara, Aditya Kumar Singha, "Comparative studies on the weldability, microstructure and tensile properties of autogeneous TIG welded AISI 430 ferritic stainless steel with and without flux," Journal of Manufacturing Processes, vol. 20, pp. 54-69, 2016.

24 J. Lippold, D. Kotecki, Welding metallurgy and weldability of stainless steel, USA: John Wiley and Sons, 2005. 